\title{
A Semiautomated Test Apparatus for Studying Partner Preference Behavior in the Rat
}

\author{
JULIE BAKKER, ${ }^{* 1}$ JAN VAN OPHEMERT,* FRITS EIJSKOOT $\dagger$ AND A. KOOS SLOB* \\ *Department of Endocrinology and Reproduction, and + Central Instrumentation Department, Faculty of Medicine \\ and Health Sciences, Erasmus University, P.O. Box 1738, 3000 DR Rotterdam, The Netherlands
}

Received 17 December 1993

\begin{abstract}
BAKKER, J., J. VAN OPHEMERT, F. EIJSKOOT AND A. K. SLOB. A semiautomated test apparatus for studying partner preference behavior in the rat. PHYSIOL BEHAV 56(3) 597-601, 1994. - A semiautomated three-compartment box (3CB) for studying partner preference behavior of rats is decribed. This apparatus automatically records the rat's time spent in each compartment, as well as the locomotor activity (i.e., the number of visits an animal pays to each compartment). Software was developed for calculating partner preference scores. Behavioral testing in the semiautomated $3 \mathrm{CB}$, which is a modification of an earlier version, is less time consuming and less laborious. Three 3 CBs can be observed simultaneously by two trained observers, and the behavioral interactions of three experimental animals with the stimulus animals can be observed and scored by hand. The use of the new apparatus was validated by studying adult partner preference behavior of neonatally ATD-treated male rats. The collected data fully corroborate previous results, obtained in the earlier version of the $3 \mathrm{CB}$, again revealing the behavioral bisexual nature of these ATD males. A new finding was the much higher locomotor activity of the ATD males compared to controls.
\end{abstract}

Neonatal ATD Rat Three-compartment box Partner preference behavior Locomotor activity

TO study motivational aspects of sexual behavior in rats, a partner preference behavior test using a three-compartment box (3CB) was developed (11). Stimulus animals could be placed in the lateral compartments of the $3 \mathrm{CB}$, either behind a wire mesh separation, which prevents sexual interaction, or tethered with a rope to the rear of one of the lateral compartments, which makes sexual interaction possible. The experimental animal could move around freely and interact with the stimulus animals or sit in the empty middle compartment. The time that the experimental animal spent in each compartment was recorded by hand, with one observer per 3CB $(1-3,11)$. Also, the various behaviors had to be scored manually. This manner of recording appeared to be time consuming and laborious.

Therefore, it was desirable to automate the $3 \mathrm{CB}$. To this end, the apparatus was changed such that the time spent in each compartment was recorded automatically. This article contains a description of the semiautomated $3 C B$ and its validation with sexually experienced male rats treated neonatally with the aromatization inhibitor ATD $(4,7,8,12)$.

\section{DESCRIPTION OF THE SEMIAUTOMATED THREE-COMPARTMENT BOX}

Figure 1 shows an artistic drawing of the semiautomated three-compartment box (wall height $40 \mathrm{~cm}$ ) and the tethering device of the stimulus animals. The box is made of gray perspex with a Plexiglas front; it has three compartments $(60 \times 30 \times 40$ $\mathrm{cm}$ each) with a small opening $(13 \times 12 \mathrm{~cm})$ in both partitions near the back. These openings can be closed by a sliding door.
Stimulus animals are tethered with a rope to the front of one of the lateral compartments, and the experimental animal freely moves around. Movement of the experimental animal from one compartment to an other is registered with position-sensitive tilt platforms placed in each opening. The height of the openings can be lowered to prevent the experimental animal from jumping over the tilt platforms. The tilt platforms are made of gray perspex and can easily be removed for cleaning purposes. The position of the tilt platform is determined using a magnetic field. Two small samarium cobalt magnets are fixed in the opposite ends of each platform. To detect the magnetic fields, sensors are placed in the bottom part of the box. Any good lab could build such an apparatus.

\section{Data Collection}

Figure 2 shows a diagram of the system. To make the data suitable for computer processing, the sensors in the bottom of the cage are connected with an interface that digitizes the sensor signals. The data of the sensor interfaces are collected by a microcontroller that can monitor three cages simultaneously (cages 1-3 or cages 4-6). The microcontroller transfers the data to a serial RS232 output, which is connected with the serial input port of a personal computer (PC). An Olivetti M24 computer attached to a Star LC-10 printer is used.

\section{Representation of the Results}

Software has been developed to draw schematically the three cages and their compartments on the monitor of the PC

\footnotetext{
'To whom requests for reprints should be addressed
} 


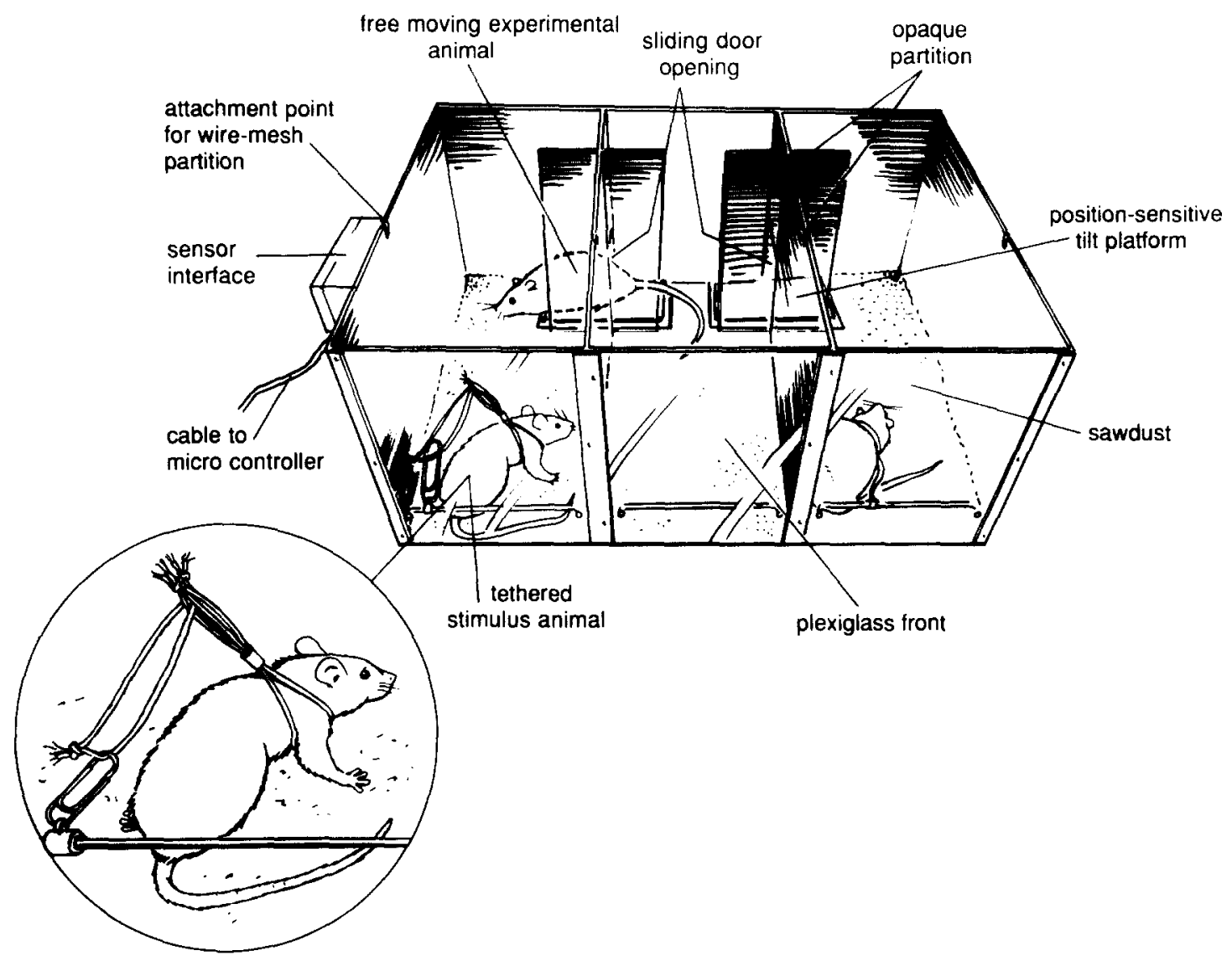

FIG. 1. Semiautomated three-ompartment box (3CB) for partner preference testing. An enlargement of one stimulus animal with the tethering device is depicted in the lower left-hand corner. (Drawing by C. de Vries, Audio Visual Center, EUR.)

(Fig. 3). When an animal moves from one compartment to another, the corresponding compartments are highlighted on the monitor. Additionally, on-line calculations are made for a preference score, and the number of visits an animal pays to each compartment are counted. A preference score is calculated by subtracting the time spent in the compartment containing the active male from the time spent in the compartment containing the estrous female (11).

\section{Testing Procedure}

Before testing begins, two stimulus animals and the experimental animal are put in the test apparatus, one in each compartment, with the sliding doors closed, for 15-min adaptation. The stimulus animals are either tethered with a rope to the front of one of the lateral compartments (sexual interaction possible) or placed behind a wire mesh separation (no sexual interaction possible). Additional data (i.e., the position of the stimulus animals) essential for calculating a preference score, the duration of testing, and a file name are fed into the computer. At the beginning of the test, the sliding doors are removed and the experimental animal can freely move around and interact with the stimulus animals or sit before the wire mesh separation. Three 3CB (three experimental animals) can be observed simultaneously by two trained observers, and the behavioral interaction of the experimental animals with the stimulus animals can be scored. The data of each test are stored on the hard disk under a chosen file name and can be printed at the end of testing for processing.

\section{SUPPORTING EXPERIMENT}

Adult partner preference behavior was studied in male rats neonatally treated with ATD (1,4,6-androstatriene-3,17-dione). ATD blocks the aromatization of testosterone to estradiol $(4,5,7,12)$. Within $2-4 \mathrm{~h}$ after birth, male rats (Wistar, RP strain) received (SC) a silastic capsule (i.d. $1.5 \mathrm{~mm}$; o.d. 2.1 $\mathrm{mm}$; length $5 \mathrm{~mm}$ ) containing ATD or cholesterol. The capsules were removed at weaning ( 21 days of age). The stimulus animals were sexually active males obtained from a commercial breeder (Harlan Sprague-Dawley Company, Zeist, The Netherlands), because at that time no locally bred adult males were available, and estrous females (locally bred; Wistar RP strain). The latter were ovariectomized and brought into behavioral estrous by injecting $20 \mu \mathrm{g}$ estradiol benzoate, $24-48 \mathrm{~h}$ before testing, followed by $1.0 \mathrm{mg}$ progesterone $3-4 \mathrm{~h}$ before testing. These hormones were dissolved in olive oil and injected SC in the neck. Behavioral testing commenced at the age of approximately 4 months. To get sexual experience, the males were first subjected to two pair tests (duration $15 \mathrm{~min}$ ) with an estrous female (all males displayed intromission behavior) and two pair tests (duration $15 \mathrm{~min}$ ) with an active male [only ATD males (five of nine) showed lordosis behavior]. After these pair tests, the male rats were subjected to three partner preference behavior tests with sexual interaction possible (tethered stimulus animals). All tests were carried out in the early portion (between $0900-1100 \mathrm{~h}$ ) of the dark phase of the light-dark cycle (lights off from 0745 to $1745 \mathrm{~h}$ ). This is the time in which ATD males were found to be sexually active both with the stimulus female 


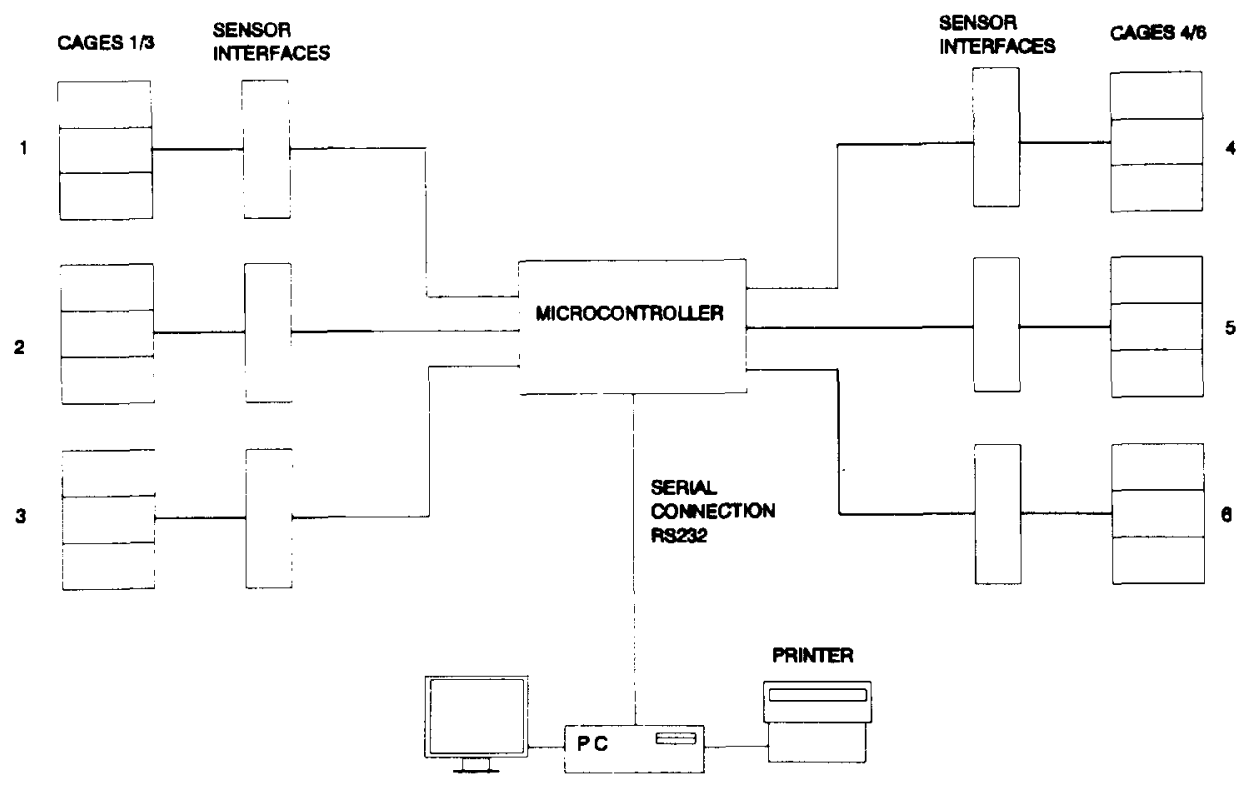

FIG. 2. System block diagram. The numbers $(1,2,3 ; 4,5,6)$ refer to the semiautomated three-compartment boxes. See the text for details.

and the stimulus male (i.e., when they were behaviorally most bisexual) $(1-3)$.

Figure 4 shows the partner preference data. Two-way analysis of variance (ANOVA) (6) on the preference scores [Fig. 4(A)] revealed a significant effect of groups, $F(1,16)=35.23, p<0.001$. The ATD male rats showed either a partner preference for the active male or no partner preference, whereas control males clearly preferred the estrous female partner. ANOVA on the time spent in the empty middle compartment [Fig. 4(B)] showed no significant differences between ATD and control male rats and no effect of testing. The time spent with the estrous female and the time spent with the active male are shown in Fig. 4(C). From looking at this figure, it is clear that ATD males spent more time with the active male and less time with the estrous female than control males.
The mean total number of visits are depicted in Fig. 4(D). ANOVA showed a significant group effect, $F(1,16)=133.2$, $p<0.001$. ATD males paid significantly more visits to the stimulus animals and the empty middle compartment than controls.

The partner preference behavior data of the present study corroborate earlier results $(1,3)$ : neonatal ATD treatment to male rats significantly affected their adult partner preference behavior. Compared to control males, such ATD males show no (or a very low) preference for the estrous female partner or a preference for the male partner. A new finding from the present study is the much higher locomotor activity of the ATD males. Although we had this impression from earlier experiments (ATD males running from one side to the other more

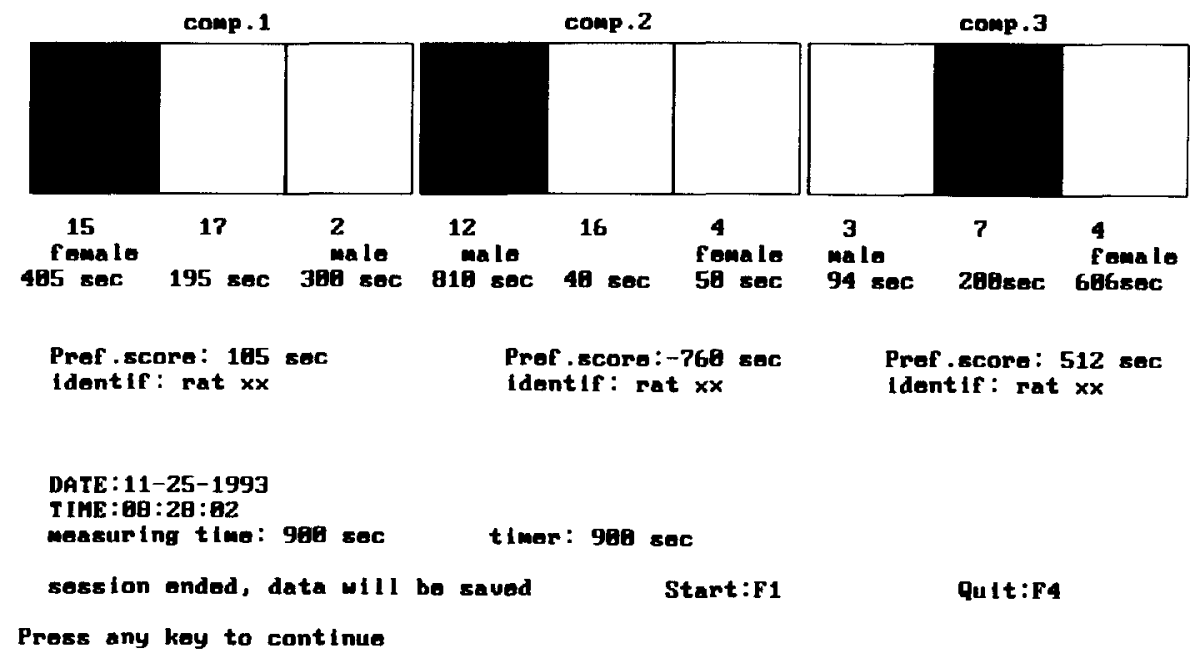

FIG. 3. Schematic drawing and other relevant information of the three semiautomated three-compartment boxes on the monitor of the personal computer. See the text for details. 

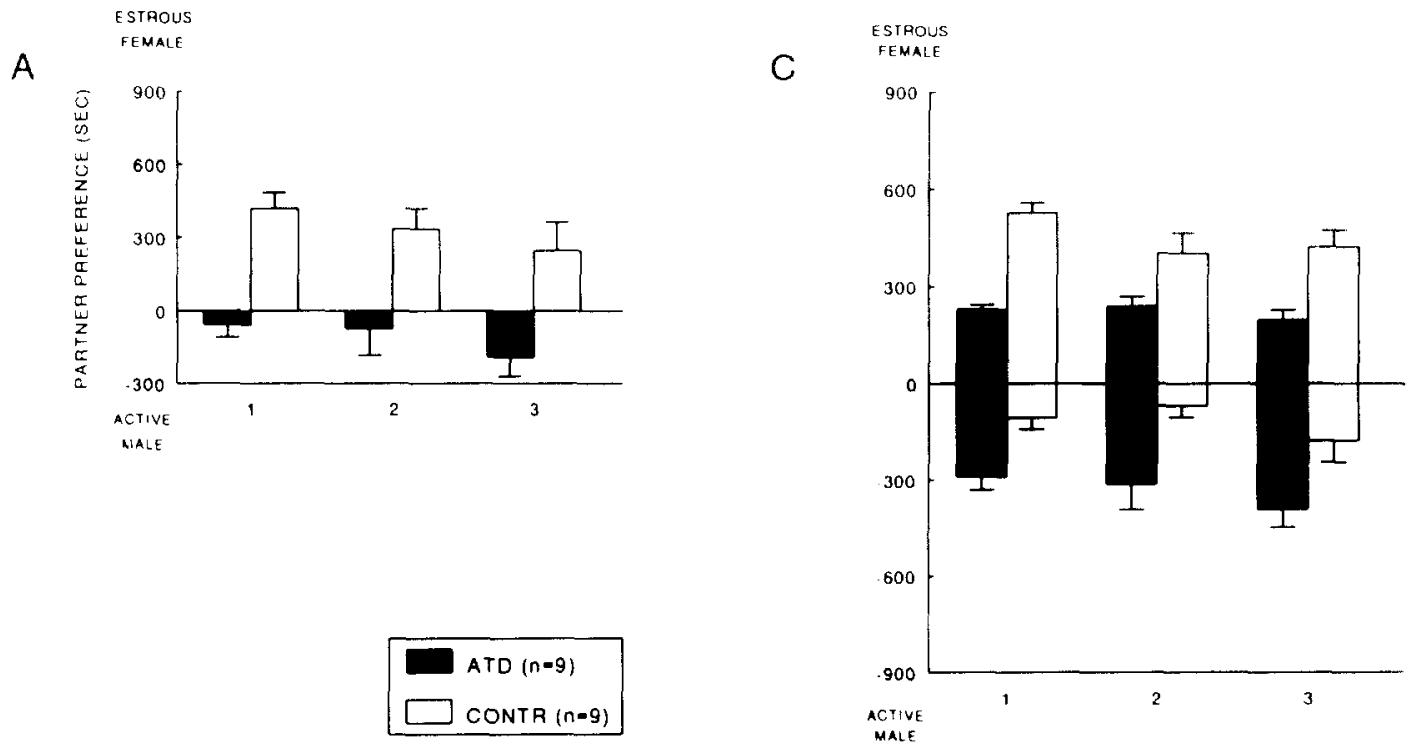

B

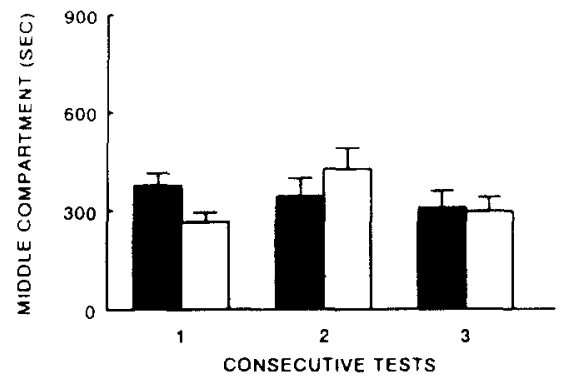

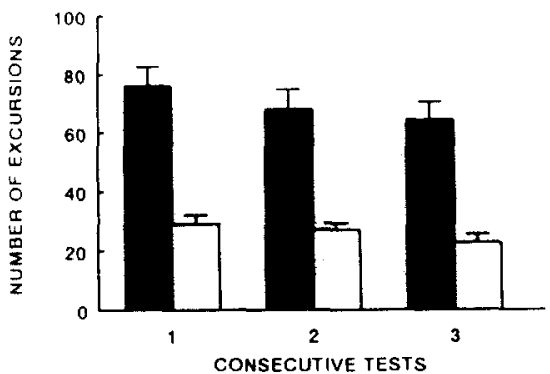

FIG. 4. Partner preference behavior of adult gonadally intact male rats, neonatally treated with the aromatization inhibitor ATD (black bars) or cholesterol (open bars). (A) Partner preference behavior, (B) time spent in the empty middle compartment, (C) time spent with the estrous female and the time spent with the active male, and (D) total number of visits to the compartments of the experimental animals. Values are means and SEM.

frequently than controls), thanks to the new semiautomated $3 \mathrm{CB}$ this supposition was confirmed and substantiated.

We do not know whether or not ATD males are generally more active or whether it is linked to the method of testing in the $3 \mathrm{CB}$ with stimulus animals. Current experiments (Verzijden and van der Helm, 1994, unpublished results) show a higher locomotor activity of ATD males independent of the stimuli offered [e.g., ovariectomized female vs. estrous female; castrated male vs. castrated testosterone-treated male; bedding only (i.e., odors) of such stimulus animals]. Sex differences are reported in locomotor activity with females showing more activity than males $(9,10)$. It is possible that the higher locomotor activity of ATD males is a manifestation of the incomplete defeminization process. More research is needed to answer this question.

In conclusion, the new semiautomated $3 \mathrm{CB}$ is a very useful apparatus for studying partner preference behavior and associated locomotor activity of rats.

\section{ACKNOWLEDGEMENTS}

The authors would like to thank Dr. P. van der Schoot for inventing the tethering device; X. E. J. van der Helm and K. Verzijden for their help with the behavioral experiment; and A. A. Brouwer, J. Storm, and J. F. Brinkman from the Central Instrumentation Department for their help with designing and modifying the $3 \mathrm{CB}$.

\section{REFERENCES}

1. Bakker, J.; Brand, T.; van Ophemert, J.; Slob, A. K. Hormonal regulation of adult partner preference behavior in neonatally ATDtreated male rats. Behav. Neurosci. 107:480-487; 1993.

2. Bakker, J.; van Ophemert, J.; Slob, A. K. Organization of partner preference and sexual behavior and its nocturnal rhythmicity in male rats. Behav. Neurosci. 107:1049-1058; 1993.

3. Brand, T.; Kroonen, J.; Mos, J.; Slob, A. K. Adult partner preference and sexual behavior of male rats affected by perinatal endocrine manipulations. Horm. Behav. 25:323-341; 1991
4. Brodie, A. M. H.; Marsh, D. A.; Wu, J. T.; Brodie, H. J. Aromatase inhibitors and their use in controlling oestrogen-dependent processes. J. Steroid Biochem. 11:107-112; 1979.

5. Kaplan, M. E.; McGinnis, M. Y. Effects of ATD on male sexual behavior and androgen receptor binding: A reexamination of the aromatization hypothesis. Horm. Behav. 23:10-26; 1989.

6. Kirk, R. E. Experimental design: Procedures for the behavioral sciences. Belmont, CA: Brooks/Cole; 1968. 
7. Lieberburg, I.; Wallach, G.; McEwen, B. S. The effects of an inhibitor of aromatization (1,4,6-androstatriene-3,17-dione) and an anti-estrogen (CI628) on in vivo formed testosterone metabolites recovered from neonatal brain tissues and purified cell nuclei. Implications for sexual differentiation of the rat brain. Brain Res. 128:176-181; 1977.

8. Schwarzel, W. C.; Kruggel, W. G.; Brodie, H. J. Studies on the mechanism of estrogen biosynthesis. VIII. The development of inhibitors of the enzyme system in human placenta. Endocrinology 92:866-880; 1973.

9. Slob, A. K.; Bogers, H.; van Stolk, M. A. Effects of gonadectomy and exogenous gonadal steroids on sex differences in open field behaviour of adult rats. Behav. Brain Res. 2:347-362; 1981 .
10. Slob, A. K.; Huizer, T.; van der Werff ten Bosch, J. J. Ontogeny of sex differences in open-field ambulation in the rat. Physiol. Behav. 37:313-315; 1986.

11. Slob, A. K.; de Klerk, L. W. L.; Brand, T. Homosexual and heterosexual partner preference in ovariectomized female rats: Effects of testosterone, estradiol and mating experience. Physiol. Behav. 41:571-576; 1987

12. Vreeburg, J. T. M.; van der Vaart, P. D. M.; van der Schoot, P. Prevention of central defeminization but not masculinization in male rats by inhibition neonatally of oestrogen biosynthesis. J. Endocrinol. 74:375-382; 1977. 